ESAIM: PROCEEDINGS AND SURVEYS, September 2014, Vol. 45, p. 328-337

J.-S. Dhersin, Editor

\title{
A COMBINED COLLOCATION AND MONTE CARLO METHOD FOR ADVECTION-DIFFUSION EQUATION OF A SOLUTE IN RANDOM POROUS MEDIA
}

\author{
Jocelyne Erhel $^{1}$, Zoubida Mghazli $^{2}$ and Mestapha Oumouni ${ }^{1,2}$
}

\begin{abstract}
In this work, we present a numerical analysis of a method which combines a deterministic and a probabilistic approaches to quantify the migration of a contaminant, under the presence of uncertainty on the permeability of the porous medium. More precisely, we consider the flow equation in a random porous medium coupled with the advection-diffusion equation. Quantities of interest are the mean spread and the mean dispersion of the solute. The means are approximated by a quadrature rule, based on a sparse grid defined by a truncated Karhunen-Loève expansion and a stochastic collocation method. For each grid point, the flow model is solved with a mixed finite element method in the physical space and the advection-diffusion equation is solved with a probabilistic Lagrangian method. The spread and the dispersion are expressed as functions of a stochastic process. A priori error estimates are established on the mean of the spread and the dispersion.

Keywords: Uncertainty quantification, elliptic PDE with random coefficients, advection-diffusion equation, collocation techniques, anisotropic sparse grids, Monte Carlo method, Euler scheme for SDE.
\end{abstract}

\section{INTRODUCTION}

Mathematical modeling and numerical simulation are important tools in the prediction of pollutant transport in groundwater and in the evaluation of potential risks of contaminants. The main constraint to the development of these models is the limited knowledge of the geological characteristics and the natural heterogeneity which implies uncertainty in the parameters and data. Stochastic approaches have been developed to deal with this uncertainty, where the permeability field is modeled as a random field $a=e^{G}$, where $G$ is a correlated Gaussian field $[2,13]$. Our objective is to quantify the migration of a contaminant by computing statistics of interest defined by the mean of the spread and of the dispersion of the solute. The permeability $a$ is discretized using a Karhunen-Loève (K-L) truncation up to a suitable and moderately large order.

The Monte Carlo method is the most widely used approach to deal with uncertainty. This method is used in $[2,4,7,12,13]$ to approximate the mean spread and dispersion.

Recently, stochastic collocation (see $[1,9,18,19]$ ), based on sparse tensor product approximation, has gained much attention since it is very effective and accurate for computing statistics from solutions of PDEs with random input data. In this paper, we use a truncated Karhunen-Loève expansion of the random data and an anisotropic sparse grid with Gaussian knots. Then we propose to approximate the mean values by a quadrature rule using this sparse grid.

${ }^{1}$ INRIA-SAGE Rennes France

${ }^{2}$ LIRNE-IMA Kenitra Morocco

(C) EDP Sciences, SMAI 2014 
The cost is proportional to the number of samples in the Monte Carlo method and to the sparse grid size in the collocation method. Each sample or grid point requires solving a flow PDE and a transport PDE; this can be done in a non intrusive and parallel way. In this paper, we approximate the steady-state flow problem by a mixed finite element method in the physical space to get the velocity field. The transport equation, which describes the concentration of the solute, is seen as a Fokker-Planck equation. A random walker method defines a stochastic process, such that the concentration is its density function. Using Itô's formula, the dispersion is expressed as a function of this stochastic process $[6,19]$. We provide new a priori error estimates on the mean spread and dispersion, taking into account all the numerical parameters.

\section{Problem Setting}

\subsection{Steady-state flow equation and transport equation}

The porous medium is assumed isotropic and the porosity is assumed equal to 1 . The Domain $D$ is a bounded box in $\mathbb{R}^{d},(d=1,2,3)$. The permeability field $a$ is modeled as a stochastic function to take into account the heterogeneity of the medium and the lack of data. Let $(\Omega, \mathcal{F}, d P)$ be a complete probability space. We consider the steady flow in a porous medium without a source data:

$$
\begin{cases}v=-a \nabla u, \operatorname{div}(v)=0 & \text { in } \Omega \times D \\ u=\gamma & \text { on } \Omega \times \partial D\end{cases}
$$

where $v$ is the velocity and $u$ is the hydraulic head. To simplify the presentation, Dirichlet boundary conditions are prescribed, with $\gamma$ sufficiently smooth. The permeability $a=e^{G}$ is $\log$-normal, where $G$ is a Gaussian field defined by its covariance function $\operatorname{cov}[G](x, z)=\sigma^{2} \exp \left\{-\frac{|x-z|^{\delta}}{l_{c}^{\delta}}\right\}$, where $\delta>0, \sigma^{2}$ is the variance and $l_{c}$ is the correlation length. For $p>1, a \in L^{p}\left(\Omega ; L^{\infty}(D)\right), \frac{1}{a_{m i n}} \in L^{p}(\Omega)$ and the problem (1) admits a unique solution $u \in L^{p}\left(\Omega ; H^{1}(D)\right)$ and $v \in L^{p}\left(\Omega ; L^{2}(D)\right)$ (see [6], for more regularity on $u$ and $v$ when $\delta=1$ ).

An inert solute is injected into the porous medium, where the kinematic dispersion is neglected and the molecular diffusion is assumed homogeneous and isotropic. Therefore, the migration of the solute is described by the advection-diffusion equation:

$$
\left\{\begin{array}{l}
\frac{\partial c}{\partial t}+v \nabla c-D_{m} \triangle c=0 \quad \text { in } \Omega \times[0, T] \times D, \\
c(\cdot, 0, x)=c_{0}(x), \quad x \in D
\end{array}\right.
$$

where $c$ is the concentration of the solute, $D_{m}$ is the diffusion coefficient and $c_{0}$ is the initial value of $c$ at $t=0$. For an injection of the solute, $c_{0}=\frac{\mathbb{1}_{B}}{|B|}$ where $B$ is a box with volume $|B|$ included in $D$. Equation (2) can be completed with Dirichlet or periodic boundary conditions on $\partial D$.

\subsection{Quantities of interest}

The main objective of our study is to compute the mean of the spread $\mathcal{S}(t)$ and the mean of the dispersion coefficient $\mathcal{D}_{t}$ (cf. [13]). First, let $I(\omega, t)=\int_{D} c(\omega, t, x) x d x$ be the center of mass of the solute distribution. Then we define $S(\omega, t)$ the spread of the solute around $I$ and $D(\omega, t)$ the dispersion coefficient as:

$$
S(\omega, t)=\int_{D} c(\omega, t, x)|x-I(\omega, t)|^{2} d x \quad \text { and } \quad D(\omega, t)=\frac{1}{2} \frac{d}{d t} S(\omega, t) .
$$

Then we are interested in the mean of $S$ and $D$ :

$$
\mathcal{S}(t)=\mathbb{E}_{\omega}[S(\cdot, t)] \quad \text { and } \quad \mathcal{D}(t)=\mathbb{E}_{\omega}[D(\cdot, t)] .
$$


In the next section, we describe the numerical methods used to approximate the mean of the spread and dispersion.

\section{NumericAl APPROACH}

\subsection{Approximation of the permeability}

We choose the Karhunen-Loève (K-L) expansion $[1,8,19]$ to approximate the field $G$ and then $a$ :

$a \approx a_{N}=e^{G_{N}}$, where $G_{N}=\mathbb{E}[G]+\sum_{m=1}^{N} \sqrt{\lambda_{m}} b_{m}(x) Y_{m}$. The set $\left\{Y_{m}\right\}_{m=1}^{N}$ are independent Gaussian random variables with zero mean and unit variance. The sequence of eigenpairs $\left(\lambda_{m}, b_{m}\right)_{m \geq 1}$ are associated to the compact self-adjoint operator $\mathcal{T}$ on $L^{2}(D)$ which is defined by:

$$
\mathcal{T}: \varphi \longmapsto \int_{D} \operatorname{cov}[G](x, \cdot) \varphi(x) d x
$$

We assume that these eigenpairs satisfy the following assumptions:

\section{Assumptions 2.1.}

- The eigenfunctions $\left(b_{m}\right)_{m>1}$ are twice continuously differentiable.

- The series $\sum_{m \geq 1} \lambda_{m}\left\|b_{m}\right\|_{\infty}^{2}$ is convergent.

Note that this assumption is satisfied in the case $\delta=1$ ( exponential covariance) or $\delta=2$ (Gaussian covariance). The field $G_{N}$ converges to $G$ in $L^{p}\left(\Omega ; L^{2}(D)\right)$ yielding the convergence of $a_{N}$ to $a$ [7]. More precisely:

$$
\left\|a-a_{N}\right\|_{L^{p}\left(\Omega ; L^{2}(D)\right)} \leq K\left(\sum_{m>N} \lambda_{m}\right)^{\frac{1}{2}} .
$$

The truncation error (5) can be bounded in terms of $N$ using the decay rate of $\lambda_{m}$ to zero. It is an algebraic or an exponential decay according to the regularity of the covariance function of $G$ (see [9] for more details).

In what follows we denote by $\mathbf{Y}=\left(Y_{1}, \ldots, Y_{N}\right)$ the random vector in $G_{N}, \Gamma=\mathbf{Y}(\Omega)=\prod_{m=1}^{N} \Gamma_{m}$, where $\Gamma_{m}=Y_{m}(\Omega)$. Let $\varrho(y)=\prod_{m=1}^{N} \rho_{m}(y)$ the density function of the vector $\mathbf{Y}$, where $\rho_{m}$ is the density of $Y_{m}$.

\subsection{Approximation of the flow problem}

We consider the parametric problem which is equivalent to the stochastic one:

$$
\begin{cases}v_{N}=-a_{N} \nabla u_{N}, \operatorname{div}\left(v_{N}\right)=0, & \text { in } \Gamma \times D \\ u_{N}=\gamma & \text { on } \Gamma \times \partial D\end{cases}
$$

The problem (6) admits a unique solution $u_{N}(\mathbf{Y}, \cdot) \in L^{p}\left(\Omega ; \mathcal{C}^{3}(D)\right)$ and $v_{N}(\mathbf{Y}, \cdot) \in L^{p}\left(\Omega ; \mathcal{C}^{2}(D)\right)[5]$. Let $\left\{\mathcal{T}_{h}\right\}_{h>0}$ be a regular triangulation of $D, \mathcal{M}_{h}$ the subspace of piecewise constants and $R T_{0}\left(\mathcal{T}_{h}\right)$ the 0 -order Raviart-Thomas subspace. Then, for each $y \in \Gamma$, we search $\left(u_{N, h}, v_{N, h}\right)$ in $\mathcal{M}_{h} \times R T_{0}\left(\mathcal{T}_{h}\right)$ solution of the problem:

$$
\begin{cases}\int_{D} a_{N}^{-1} v_{N, h} w_{h} d x-\int_{D} u_{N, h} \operatorname{div}\left(w_{h}\right)=-\int_{\partial D} \gamma w_{h} \cdot \vec{n} d l & \forall w_{h} \in R T_{0}\left(\mathcal{T}_{h}\right), \\ \int_{D} \operatorname{div}\left(v_{N, h}\right) \mu_{h}=0, & \forall \mu_{h} \in \mathcal{M}_{h} .\end{cases}
$$

Problem (7) is well-posed [3] for each (a.e) $y \in \Gamma$, Moreover the velocity $v_{N, h} \in L^{p}\left(\Omega ; L^{\infty}(D)\right)$. The solution $\left(u_{N}, v_{N}\right)$ is smooth with respect to $x$, then by the deterministic error estimate [3], there exists a constant $C$ independent of $h, v_{N}$ and $u_{N}$, such that

$$
\left\|u_{N}-u_{N, h}\right\|_{L^{p}\left(\Omega ; L^{2}(D)\right)}+\left\|v_{N}-v_{N, h}\right\|_{L^{p}(\Omega ; H(\operatorname{div}, D))} \leq C h\left(\left\|v_{N}\right\|_{L^{p}\left(\Omega ; H^{1}(D)\right)}+\left\|u_{N}\right\|_{L^{p}\left(\Omega ; H^{1}(D)\right)}\right) .
$$




\subsection{Approximation of the transport problem}

The quantities of interest are given by an integral of $c$ as in (3). Then, in order to avoid to approximate $c$ at each point in $D$ and to avoid numerical diffusion, a Lagrangian method is preferred to an Eulerian method [12]. This method consists in simulating a cloud of particles in the physical domain: when (2) is considered on $\mathbb{R}^{d}, c$ is a law of the process describing the position of the particles. In practice, $D$ is chosen very big versus $B$ and a very small amount of the solute gets at $\partial D\left(c_{\partial D} \approx 0\right)$. It is safe to replace $(2)$ by:

$$
\left\{\begin{array}{l}
\frac{\partial c}{\partial t}(\omega, t, x)+v(\omega, x) \nabla c(\omega, t, x)-D_{m} \triangle c(\omega, t, x)=0 \quad \text { in } \Omega \times[0, T] \times \mathbb{R}^{d}, \\
c(\omega, 0, x)=c_{0}(x) \quad x \in \mathbb{R}^{d},
\end{array}\right.
$$

where $v$ is extended continuously by zero outside a neighborhood of $D$. The unique solution of (9) belongs to $L^{p}\left(\Omega ; L^{\infty}\left([0, T] \times \mathbb{R}^{d}\right)\right)$ [7]. For each $\omega \in \Omega, c(\omega, \cdot)$ is the density function of $Y_{t}(\omega, \cdot)$, solution of the following SDE (cf $[10])$ :

$$
\left\{\begin{array}{l}
d Y_{t}(\omega, \theta)=v\left(\omega, Y_{t}(\omega, \theta)\right) d t+\sqrt{2 D_{m}} d W_{t}(\theta) \\
Y_{0}(\omega, \theta)=\zeta(\theta)
\end{array}\right.
$$

where $\left(W_{t}\right)$ is a $d$-dimensional Brownian motion on another probability space $(\Theta, \mathfrak{A}, \mathcal{P})$ and $\zeta$ is a random variable $W_{t}$-independent, which admits $c_{0}$ as a density. We assume that it satisfies $\mathbb{E}\left[|\zeta|^{q}\right]<\infty$ for $q \geq 1$. Thus, the spread is given by the variance of $Y_{t}$ :

$$
S(\omega, t)=\mathbb{E}_{\theta}\left[\left|Y_{t}-\mathbb{E}_{\theta}\left[Y_{t}\right]\right|^{2}\right]=\mathbb{E}_{\theta}\left[\left|Y_{t}\right|^{2}\right]-\left|\mathbb{E}_{\theta}\left[Y_{t}\right]\right|^{2} .
$$

The dispersion $D(\omega, t)$ was estimated by a Finite Difference approximation in $[4,11,12,16]$, but the result is very sensitive to the step taken. Here we use an explicit formula to compute $D(\omega, t)$ (see $[6,19]$ ). First, we have $\frac{d}{d t}\left|\mathbb{E}_{\theta}\left[Y_{t}\right]\right|^{2}=2<\mathbb{E}_{\theta}\left[Y_{t}\right], \mathbb{E}_{\theta}\left[v\left(Y_{t}\right)\right]>$; by Itô's formula $d\left|Y_{t}\right|^{2}=2\left(<Y_{t}, v\left(Y_{t}\right)>+\operatorname{trace}\left(D_{m}\right)\right) d t+2 Y_{t} \sqrt{2 D_{m}} d W_{t} ;$ so $\frac{d}{d t} \mathbb{E}\left[\left|Y_{t}\right|^{2}\right]=2\left(\mathbb{E}_{\theta}\left[<Y_{t}, v\left(Y_{t}\right)>\right]+\operatorname{trace}\left(D_{m}\right)\right)$. Hence we obtain:

$$
D(\omega, t)=\mathbb{E}_{\theta}\left[<Y_{t}, v\left(Y_{t}\right)>\right]-<\mathbb{E}_{\theta}\left[Y_{t}\right], \mathbb{E}_{\theta}\left[v\left(Y_{t}\right)\right]>+\operatorname{trace}\left(D_{m}\right) .
$$

Here, $a$ is approximated by $a_{N}(\mathbf{Y},$.$) and v$ by $v_{N}(\mathbf{Y},$.$) . Then, c$ is approximated by $c_{N}(\mathbf{Y},$.$) which is a density$ function of a process $X_{t}$. Moreover, since the differential operator is continuous, then by Doob Dynkin's lemma, $X_{t}$ is parameterized by $\mathbf{Y}$ as $X_{t}(\omega, \cdot)=X_{t}(\mathbf{Y}(\omega), \cdot)$. For $y \in \Gamma, X_{t}(y)$ is solution of the following SDE:

$$
\left\{\begin{array}{l}
d X_{t}(y, \theta)=v_{N}\left(y, X_{t}(y, \theta)\right) d t+\sqrt{2 D_{m}} d W_{t}(\theta) \\
X_{0}(y, \theta)=\zeta(\theta)
\end{array}\right.
$$

The spread (11) and the dispersion term $D(\omega, t)-\operatorname{trace}\left(D_{m}\right)$ in $(12)$ are approximated by

$$
\begin{aligned}
S_{N}(y, t) & =\mathbb{E}_{\theta}\left[\left|X_{t}(y, \cdot)\right|^{2}\right]-\left|\mathbb{E}_{\theta}\left[X_{t}(y, \cdot)\right]\right|^{2} \\
D_{N}(y, t) & =\mathbb{E}_{\theta}\left[<X_{t}(y, \cdot), v_{N}\left(y, X_{t}(y, \cdot)\right)>\right]-<\mathbb{E}_{\theta}\left[X_{t}(y, \cdot)\right], \mathbb{E}_{\theta}\left[v_{N}\left(y, X_{t}(y, \cdot)\right)\right]>
\end{aligned}
$$

For a given $y \in \Gamma$, we solve (7) to compute $v_{N, h}(y, \cdot)$ and we use the Euler scheme to discretize (13):

$$
\left\{\begin{array}{l}
X_{n, h}\left(y, \theta, t_{l+1}\right)=X_{n, h}\left(y, \theta, t_{l}\right)+v_{N, h}\left(y, X_{n, h}\left(y, \theta, t_{l}\right)\right) d t+\sqrt{2 D_{m} d t} \xi_{l+1}(\theta) \\
X_{n, h}(y, \theta, 0)=\zeta(\theta)
\end{array}\right.
$$

where $\left\{\xi_{l+1}\right\}_{l=0}^{n}$ are independent gaussian variables with zero mean and unit variance and $d t=\frac{T}{n}$. We choose $M$ realizations $\left\{X_{n, h}^{k}(y, t)\right\}_{k=1}^{M}$ for $X_{n, h}(y, t)$, then we approximate $S_{N}(y, t)(14)$ by MC sampling:

$$
S_{N, M}(y, t)=\frac{1}{M} \sum_{k=1}^{M}\left|X_{n, h}^{k}(y, t)\right|^{2}-\left|\frac{1}{M} \sum_{k=1}^{M} X_{n, h}^{k}(y, t)\right|^{2}
$$


and the dispersion term $D_{N}(y, t)$ defined in (15) by:

$$
D_{N, M}(y, t)=\frac{1}{M} \sum_{k=1}^{M}<X_{n, h}^{k}(y, t), v_{N, h}\left(y, X_{n, h}^{k}(y, t)\right)>-\frac{1}{M^{2}} \sum_{k, l=1}^{M}<X_{n, h}^{k}(y, t), v_{N, h}\left(y, X_{n, h}^{l}(y, t)\right)>.
$$

\subsection{Approximation of the mean spread and mean dispersion}

The standard sparse grid quadrature is based on the Smolyak algorithm [20]. The anisotropic sparse grid quadrature is a generalization, with weights in each direction $[18,19]$. These methods provide an effective way to approach the integral of a multivariate function $\psi$ defined by

$$
I(\psi)=\int_{\Gamma} \psi(y) \varrho(y) d y
$$

We follow closely $[18,19]$ to define this technique. Let $\mathbf{i}$ a multi-index of $\mathbb{N}_{+}^{N}$ and $\mathcal{X}^{\mathbf{i}}=\prod_{m=1}^{N} X^{i_{m}} \subset \Gamma$ a tensor grid, where each $X^{i_{m}}$ is the set of Gauss quadrature knots. Let $\alpha_{1}, \ldots, \alpha_{N} \in \mathbb{R}^{+}, N$ weights for the different stochastic dimensions and $\underline{\alpha}=\min _{m} \alpha_{m}$. We consider a level $w \geq 0$ and we define the multi-index set:

$$
Z_{\alpha}(w, N)=\left\{\mathbf{i} \in \mathbb{N}^{N}, w \underline{\alpha}<\sum_{m=1}^{N} \alpha_{m} i_{m} \leq w \underline{\alpha}+\sum_{m=1}^{N} \alpha_{m}\right\} .
$$

Then, we define an anisotropic sparse grid as a subset of $\Gamma$ by

$$
\mathcal{H}_{\alpha}(w, N)=\bigcup_{\mathbf{i} \in Z_{\alpha}(w, N)} \mathcal{X}^{\mathbf{i}}=\left\{y_{j}, j=1 \ldots, \eta\right\}
$$

where $\eta$ is the cardinal of $\mathcal{H}_{\alpha}(w, N)$. The quadrature rule $\mathcal{Q}_{\alpha}(w, N)$ with level $w$ is defined by:

$$
I(\psi) \approx \mathcal{Q}_{\alpha}(w, N) \psi=\sum_{j=0}^{\eta} \psi\left(y_{j}\right) \mu_{j},
$$

where $\mu_{j}$ are the quadrature weights associated to each point $y_{j}$ of the method and defined by Gauss quadrature rules $[18,19]$ in each direction. When the integrand $\psi$ is analytic on a bounded set $\Gamma$ or an entire function on an unbounded set, the anisotropic sparse grid method converges at least with an algebraic convergence if each $\alpha_{m}$ is the exponential rate of the Lagrange polynomial approximation of $\psi$ with respect to direction $m[18,19]$ :

$$
\left|I(\psi)-\mathcal{Q}_{\alpha}(w, N) \psi\right| \leq C(N, \alpha) \eta^{-\tau},
$$

where $\tau=\frac{\underline{\alpha}}{\log \left(1+\sum_{m=1}^{N} \frac{\underline{\alpha}}{\alpha_{m}}\right)}$ is the convergence rate.

The mean spread and the mean dispersion can be computed using (17) and (18) by the following integrals:

$$
\mathcal{S}(t) \approx \int_{\Gamma} S_{N, M}(y, t) \varrho(y) d y \text { and } \mathcal{D}(t) \approx \int_{\Gamma} D_{N, M}(y, t) \varrho(y) d y+\operatorname{trace}\left(D_{m}\right) .
$$

We simply apply the quadrature rule (19) to approximate the integrals (21) and get:

$$
\begin{aligned}
\mathcal{S}_{N, M, \eta}(t) & =\sum_{j=1}^{\eta} S_{N, M}\left(y_{j}, t\right) \mu_{j}, \\
\mathcal{D}_{N, M, \eta}(t) & =\sum_{j=1}^{\eta} D_{N, M}\left(y_{j}, t\right) \mu_{j}+\operatorname{trace}\left(D_{m}\right) .
\end{aligned}
$$

The cost of the method is thus proportional to the size of the sparse grid. This size is related to the dimension $N$, which in turn depends on the correlation length $l_{c}$ (see, $\left.[18,19]\right)$.

In the next section, we give an error analysis of the approach, especially, the errors on $\mathcal{S}(T)$ and $\mathcal{D}(T)$ when we approximate them respectively by (22) and (23). 


\section{ERror ANALYSis of THE APPROACH}

We consider $\mathcal{C}_{\text {pol }}^{l}\left(\mathbb{R}^{d}\right)$, the space of functions which have a polynomial growth with their derivatives up to $l$. Let $p>1, q \geq 1$ and $l \geq 0$. Let $\phi$ in $\mathcal{C}_{\text {pol }}^{\infty}\left(\mathbb{R}^{d}\right)$ and $g$ in $L^{p}\left(\Omega ; \mathcal{C}_{\text {pol }}^{l}\left(\mathbb{R}^{d}\right)\right)$. We assume that $g=g_{1}(x) g_{2}(\omega, x)$ where $g_{1} \in \mathcal{C}_{\text {pol }}^{l}\left(\mathbb{R}^{d}\right), g_{2} \in L^{p}\left(\Omega ; \mathcal{C}_{b}^{l}\left(\mathbb{R}^{d}\right)\right)$ and $g_{2}$ can be approximated by an analytic function $g_{2 N}$ on $\Gamma \times \mathbb{R}^{d}$ such that $g_{2 N}(\mathbf{Y},$.$) converges to g_{2}(\mathbf{Y},$.$) in L^{p}\left(\Omega ; \mathcal{C}_{b}^{l}\left(\mathbb{R}^{d}\right)\right)$ as follow:

$$
\left\|g_{2}-g_{2 N}\right\|_{L^{p}\left(\Omega ; \mathcal{C}_{b}^{l}\left(\mathbb{R}^{d}\right)\right)} \leq K_{2}\left(\sum_{m>N} \lambda_{m}\right)^{\frac{1}{2}} .
$$

The objective is to establish total weak errors in $\Omega \times \Theta$, when the mean on $\Omega$ is computed by the quadrature formula $\mathcal{Q}_{\alpha}(w, N)$ defined in (19) and the mean on $\Theta$ by Monte Carlo sampling defined in $(17,18)$.

Definition 3.1. We define the total weak error in $\Omega \times \Theta$ by:

$$
\mathcal{E} r(T):=\mathbb{E}_{\omega}\left[\phi\left(\mathbb{E}_{\theta}\left[g\left(Y_{T}\right)\right]\right)\right]-\mathcal{Q}_{\alpha}(w, N) \phi\left(\frac{1}{M} \sum_{k=1}^{M} g_{N}\left(X_{n, h}^{k}(T)\right)\right),
$$

where $\mathcal{Q}_{\alpha}(w, N)$ is the quadrature formula (19) and $g_{N}=g_{1} g_{2_{N}}$, with $g_{1}$ and $g_{2_{N}}$ as defined above.

An error estimate on $\mathcal{S}(T)$ follows with $g(\omega, x)=|x|^{2}$ and $\phi(x)=x$ and $g(\omega, x)=x, \phi(x)=|x|^{2}$. The error estimate on $\mathcal{D}(T)$ follows after dividing the error in two parts with the choice $g(\omega, x)=<x, v(\omega, x)\rangle, \phi(x)=x$, $g_{2 N}(y, x)=v_{N}(y, x)$ and $\phi(x)=x$ side by side. We note that the assumption on $g$ is fulfilled with this latter choice, when the covariance function of $G$ is regular, for example an exponential or Gaussian covariance.

\subsection{Truncation error}

In this section, we give a bound of the truncation error resulting from the truncation of the parameter $a \approx a_{N}$.

Proposition 3.1. There exists $K_{1}$ a positive constant such that:

$$
\left\|v-v_{N}\right\|_{L^{p}\left(\Omega, L^{2}(D)\right)} \leq K_{1}\left\|a-a_{N}\right\|_{L^{2 p}\left(\Omega ; L^{2}(D)\right)} .
$$

Proof: For each $\omega \in \Omega$ and $\mu \in H_{0}^{1}(D)$, we have: $\int_{D} a \nabla u \nabla \mu d x=\int_{D} a_{N} \nabla u_{N} \nabla \mu d x=0$, then

$$
\begin{aligned}
\int_{D} a \nabla\left(u-u_{N}\right) \nabla \mu d x & =\int_{D} a \nabla u \nabla \mu d x+\int_{D}\left(a_{N}-a\right) \nabla u_{N} \nabla \mu d x-\int_{D} a_{N} \nabla u_{N} \nabla \mu d x \\
& =\int_{D}\left(a_{N}-a\right) \nabla u_{N} \nabla \mu d x \leq\left\|u_{N}\right\|_{\mathcal{C}^{1}(\bar{D})}\left\|a-a_{N}\right\|_{L^{2}(D)}\|\nabla \mu\|_{L^{2}(D)},
\end{aligned}
$$

taking $\mu=u-u_{N}$, we get

$$
\left\|u-u_{N}\right\|_{H_{0}^{1}(D)} \leq \frac{\left\|u_{N}\right\|_{\mathcal{C}^{1}(\bar{D})}}{a_{\min }(\omega)}\left\|a-a_{N}\right\|_{L^{2}(D)} \leq \frac{P\left(\|a\|_{\infty},\|\gamma\|_{\mathcal{C}^{0}(\bar{D})}\right)}{a_{\min }(\omega)}\left\|a-a_{N}\right\|_{L^{2}(D)}
$$

since $\left\|u_{N}\right\|_{\mathcal{C}^{1}(\bar{D})} \leq P\left(\left\|a_{N}\right\|_{\infty},\|\gamma\|_{\mathcal{C}^{0}(\bar{D})}\right)$ where $P$ is a polynomial (see, [5]) and $\left\|a_{N}\right\|_{\infty} \leq\|a\|_{\infty}$.

On the other hand, we have for each $\omega \in \Omega$,

$$
\begin{aligned}
\left\|v-v_{N}\right\|_{L^{2}(D)} & =\left\|\left(a-a_{N}\right) \nabla u+a_{N} \nabla\left(u-u_{N}\right)\right\|_{L^{2}(D)} \\
& \leq\left\|a-a_{N}\right\|_{L^{2}(D)}\|u\|_{\mathcal{C}^{1}(\bar{D})}+\left\|u-u_{N}\right\|_{H_{0}^{1}(D)}\left\|a_{N}\right\|_{\infty} \leq \tilde{C}(\omega)\left\|a-a_{N}\right\|_{L^{2}(D)}
\end{aligned}
$$

where $\tilde{C}(\omega)=\|u\|_{\mathcal{C}^{1}(\bar{D})}+\frac{P\left(\|a\|_{\infty},\|\gamma\|_{\mathcal{C}^{0}(\bar{D})}\right)}{a_{\min }}\|a\|_{\infty}$. The estimate is concluded by taking the norm in $L^{p}(\Omega)$ and Hölder inequality, with $K_{1}=\|\tilde{C}(.)\|_{L^{2 p}(\Omega)}$ which is finite since $\|a\|_{\infty}$ and $\frac{1}{a_{m i n}}$ belong to $L^{p}(\Omega)$ (see, $[5]$ ).

The following bound is useful: it shows that the mean of any function of $Y_{t}$ with polynomial growth is finite. 
Lemma 3.1. There exists $C_{p, q, T}>0$ such that:

$$
\mathbb{E}_{\omega}\left[\left|\mathbb{E}_{\theta}\left[\left|Y_{t}\right|^{p}\right]\right|^{q}\right] \leq C_{p, q, T} \mathbb{E}_{\theta}\left[\left|Y_{0}\right|^{p}\right] .
$$

Proof: it is straightforward, since $Y_{t}=Y_{0}+\int_{0}^{t} v\left(., Y_{s}\right) d s+\sqrt{2 D_{m}} W_{t}$, and $v \in L^{p}\left(\Omega ; L^{\infty}\left(\mathbb{R}^{d}\right)\right)$. A similar bound holds for the process $X_{t}$ and its approximation $X_{n, h}$ given in (16) regardless of $N, h$ and $n$

The following result provides an estimate of the truncation error on the quantities of interest.

Proposition 3.2. For any $g$ in $L^{p}\left(\Omega ; \mathcal{C}_{\text {pol }}^{l}\left(\mathbb{R}^{d}\right)\right)$, there exists $C>0$, independent of $N$ such that:

$$
\left.\mathbb{E}_{\omega}\left[\mathbb{E}_{\theta}\left[g\left(\cdot, Y_{T}\right)-g\left(\cdot, X_{T}\right)\right)\right]\right] \leq C\left(\sum_{m>N} \lambda_{m}\right)^{\frac{1}{2}} .
$$

Proof: Let $u$ be a solution of the Kolmogorov backward equation associated to (13):

$$
\left\{\begin{array}{l}
\partial_{t} u(\omega, t, x)+v_{N}(\omega, x) \cdot \nabla u(\omega, t, x)+D_{m} \Delta u(\omega, t, x)=0 \quad 0 \leq t<T \\
u(\omega, T, x)=g(\omega, x) .
\end{array}\right.
$$

Since the trajectories of $v_{N}$ and $g$ are $\mathcal{C}_{\text {pol }}^{2}\left(\mathbb{R}^{d}\right)$, the trajectories of $u$ belong to $\mathcal{C}^{1}\left(\left[0, T\left[, \mathcal{C}_{\text {pol }}^{4}\left(\mathbb{R}^{d}\right)\right) \cap \mathcal{C}^{1}\left([0, T], \mathcal{C}_{\text {pol }}^{2}\left(\mathbb{R}^{d}\right)\right)\right.\right.$ (see [15]) and are given by $u(t, x)=\mathbb{E}_{\theta}\left[g\left(X_{T}\right) \mid X_{t}=x\right]$ (Feynman-Kac formula, see [14]). In particular $u\left(0, Y_{0}\right)=u\left(0, X_{0}\right)=\mathbb{E}_{\theta}\left[g\left(X_{T}\right)\right]$. We define the following weak error:

$$
e_{T}=\mathbb{E}_{\theta}\left[g\left(Y_{T}\right)\right]-\mathbb{E}_{\theta}\left[g\left(X_{T}\right)\right]=\mathbb{E}_{\theta}\left[u\left(T, Y_{T}\right)\right]-\mathbb{E}_{\theta}\left[u\left(0, Y_{0}\right)\right]
$$

Using Itô's formula, we have:

$$
u\left(T, Y_{T}\right)-u\left(0, Y_{0}\right)=\int_{0}^{T}\left(\frac{\partial u}{\partial t}+v\left(Y_{s}\right) \cdot \nabla u+D_{m} \triangle u\right)\left(s, Y_{s}\right) d s+\int_{0}^{T} \sqrt{2 D_{m}} \nabla u\left(s, Y_{s}\right) d W s,
$$

Therefore, $e_{T}=\int_{0}^{T} \mathbb{E}_{\theta}\left[\left(\frac{\partial u}{\partial t}+v\left(Y_{s}\right) \cdot \nabla u+D_{m} \triangle u\right)\left(s, Y_{s}\right)\right] d s$. Using (30) at $\left(s, Y_{s}\right)$, we obtain:

$$
\left.e_{T}=\int_{0}^{T} \mathbb{E}_{\theta}\left[\left(v\left(Y_{s}\right)-v_{N}\left(Y_{s}\right)\right)\right) \nabla u\left(s, Y_{s}\right)\right] d s \leq \sup _{0 \leq t \leq T}\left\|\nabla u\left(t, Y_{t}\right)\right\|_{L^{2}(\theta)} \int_{0}^{T}\left\|v\left(Y_{s}\right)-v_{N}\left(Y_{s}\right)\right\|_{L^{2}(\theta)} d s
$$

The solution $c$ of (9) is the density function of $Y_{t}$ on $\Theta$ a.e in $\Omega$, then,

$$
\left\|v_{N}\left(Y_{t}\right)-v\left(Y_{t}\right)\right\|_{L^{2}(\Theta)}^{2}=\int_{\mathbb{R}^{d}}\left|v(x)-v_{N}(x)\right|^{2} c(\cdot, t, x) d x \leq \sup _{0 \leq t \leq T} \sup _{x \in \mathbb{R}^{d}} c(\cdot, t, x)\left\|v-v_{N}\right\|_{L^{2}\left(\mathbb{R}^{d}\right)}^{2} .
$$

Combining this with (31), we obtain:

$$
e_{T} \leq T \sup _{0 \leq t \leq T}\left\|\nabla u\left(t, Y_{t}\right)\right\|_{L^{2}(\Theta)} \sup _{0 \leq t \leq T} \sup _{x \in \mathbb{R}^{d}} \sqrt{c(\cdot, t, x)}\left\|v-v_{N}\right\|_{L^{2}\left(\mathbb{R}^{d}\right)} .
$$

Taking the expectation on $\Omega$ and using the Hölder inequality, we get:

$$
\mathbb{E}_{\omega}\left[e_{T}\right] \leq T C_{1}\left\|v-v_{N}\right\|_{L^{2}\left(\Omega, L^{2}\left(\mathbb{R}^{d}\right)\right)},
$$

where $C_{1}=\left\|\sup _{t \leq T} \nabla u\left(t, Y_{t}\right)\right\|_{L^{4}\left(\Omega ; L^{2}(\Theta)\right)}\left\|\sup _{t \leq T, x \in \mathbb{R}^{d}} \sqrt{c(\cdot, t, x)}\right\|_{L^{4}(\Omega)} . \mathrm{Using}(28),\left\|\sup _{t \leq T}\right\| \nabla u\left(t, Y_{t}\right)\left\|_{L^{2}(\Theta)}\right\|_{L^{4}\left(\Omega ; L^{2}(\Theta)\right)}$ is finite. Also $c \in L^{q}\left(\Omega ; L^{\infty}\left([0, T] \times \mathbb{R}^{d}\right)\right)[7],\left\|\sup _{t \leq T, x \in \mathbb{R}^{d}} \sqrt{c(\cdot, t, x)}\right\|_{L^{4}(\Omega)}$ is finite. Thus $C_{1}$ is finite. We conclude the proof with the bounds (26) and (5), and get $C=K K_{1} T C_{1}$. 


\subsection{Temporal and spatial discretization error}

Here, we give a bound of the weak error $\mathbb{E}_{\omega}\left[\mathbb{E}_{\theta}\left[g\left(X_{T}\right)\right]-\mathbb{E}_{\theta}\left[g\left(X_{n, h}\right)\right]\right]$. It can be divided into tow terms. The first one related to time discretization is classical when the drift is $\mathcal{C}^{2}$ [17]. The second term concerns the space error and we show that it has the order $h$. Let $X_{n}(t)$ be an approximation by Euler scheme of the process $X_{t}$ and $X_{n, h}(t)$ its perturbation as given in (16).

Proposition 3.3. There exists a constant $C(T, g)>0$ independent of $d t$ and $h$ such that:

$$
\mathbb{E}_{\omega}\left[\mathbb{E}_{\theta}\left[g\left(\cdot, X_{T}\right)\right]-\mathbb{E}_{\theta}\left[g\left(\cdot, X_{n, h}(T)\right)\right]\right] \leq C(T, g)(d t+h) .
$$

Proof: Let $u$ solution of (30), then:

$$
\mathbb{E}_{\omega}\left[\mathbb{E}_{\theta}\left[g\left(\cdot, X_{n, h}(T)\right)\right]-\mathbb{E}_{\theta}\left[g\left(\cdot, X_{T}\right)\right]\right]=\mathbb{E}_{\omega}\left[\mathbb{E}_{\theta}\left[u\left(T, X_{n, h}(T)\right)\right]-\mathbb{E}_{\theta}\left[u\left(0, X_{0}\right)\right]\right]=\sum_{l=1}^{n} \mathbb{E}_{\omega}\left[\mathbb{E}_{\theta}\left[e_{l}\right]\right]
$$

where we set $e_{l}=u\left(t_{l+1}, X_{n, h}\left(t_{l+1}\right)\right)-u\left(t_{l}, X_{n, h}\left(t_{l}\right)\right)$. Using Itô's formula, we have:

$$
e_{l}=\int_{t_{l}}^{t_{l+1}}\left(\frac{\partial u}{\partial t}+v_{N, h}\left(X_{n, h}\left(t_{l}\right)\right) \nabla u+D_{m} \triangle u\right)\left(s, X_{n, h}(s)\right) d s+\int_{t_{l}}^{t_{l+1}} \sqrt{2 D_{m}} \nabla u\left(s, X_{n, h}(s)\right) d W s,
$$

we conclude that, $\mathbb{E}_{\theta}\left[e_{l}\right]=\int_{t_{l}}^{t_{l+1}} \mathbb{E}_{\theta}\left[\left(\frac{\partial u}{\partial t}+v_{N, h}\left(X_{n, h}\left(t_{l}\right)\right) \nabla u+D_{m} \triangle u\right)\left(s, X_{n, h}\right)\right] d s$.

Using (30) at point $\left(s, X_{n, h}(s)\right)$ and then taking the mean on $\Omega$, we obtain:

$$
\mathbb{E}_{\omega}\left[\mathbb{E}_{\theta}\left[e_{l}\right]\right]=\int_{t_{l}}^{t_{l+1}} \mathbb{E}_{\theta}\left[\left(v_{N, h}\left(X_{n, h}\left(t_{l}\right)\right)-v_{N}\left(X_{n, h}(s)\right)\right) \nabla u\left(s, X_{n, h}(s)\right)\right] d s=\mathbb{E}_{\omega}\left[J_{1}\right]+\mathbb{E}_{\omega}\left[J_{2}\right],
$$

where we define $J_{1}:=\int_{t_{l}}^{t_{l+1}} \mathbb{E}_{\theta}\left[\left(v_{N, h}\left(X_{n, h}\left(t_{l}\right)\right)-v_{N}\left(X_{n, h}\left(t_{l}\right)\right)\right) \nabla u\left(s, X_{n, h}(s)\right)\right] d s$ and the second term by $J_{2}:=\int_{t_{l}}^{t_{l+1}} \mathbb{E}_{\theta}\left[\left(v_{N}\left(X_{n, h}\left(t_{l}\right)\right)-v_{N}\left(X_{n, h}(s)\right)\right) \nabla u\left(s, X_{n, h}(s)\right)\right] d s$. For each $l=2, \ldots, n+1$, let $c_{n, h}\left(\omega, t_{l}, x\right)$ the density of $X_{n, h}\left(\omega, t_{l},.\right)$. It belongs to $\mathcal{C}_{0}^{\infty}\left(\mathbb{R}^{d}\right)$, since it is given by the convolution of the density of the variable $X_{n, h}\left(\omega, t_{l},.\right)+v_{h}\left(X_{n, h}\left(\omega, t_{l},.\right) d t\right.$ with the density of $\sqrt{2 D_{m} d t} \xi_{l+1}$, and by induction we get:

$c_{n, h}\left(\omega, t_{l}, x\right) \leq \tilde{K}\left\|c_{0}\right\|_{\infty}\left(1+\|v\|_{\infty} l d t\right)$, where $\tilde{K}>0$ and independent of $h$ and $n$. The term $J_{1}$ satisfies:

$$
\begin{aligned}
J_{1} & \leq \int_{t_{l}}^{t_{l+1}}\left\|v_{N, h}\left(X_{n, h}\left(t_{l}\right)\right)-v_{N}\left(X_{n, h}\left(t_{l}\right)\right)\right\|_{L^{2}(\Theta)}\left\|\nabla u\left(s, X_{n, h}(s)\right)\right\|_{L^{2}(\Theta)} d s \\
& \leq \int_{t_{l}}^{t_{l+1}}\left\|\nabla u\left(s, X_{n, h}(s)\right)\right\|_{L^{2}(\Theta)} d s\left(\int_{\mathbb{R}^{d}}\left|v_{N, h}(x)-v_{N}(x)\right|^{2} c_{n, h}\left(t_{l}, x\right) d x\right)^{\frac{1}{2}} \\
& \leq d t \sup _{x \in \mathbb{R}^{d}} \sup _{l \leq n+1} \sqrt{c_{n, h}\left(t_{l}, x\right)} \sup _{t \leq T}\left\|\nabla u\left(t, X_{n}(t)\right)\right\|_{L^{2}(\Theta)}\left\|v_{N}-v_{N, h}\right\|_{L^{2}\left(\mathbb{R}^{d}\right)}
\end{aligned}
$$

Taking the mean on $\Omega$, we get:

$$
\mathbb{E}_{\omega}\left[J_{1}\right] \leq C_{1}(d t)\left\|v_{N}-v_{N, h}\right\|_{L^{2}\left(\Omega ; L^{2}\left(\mathbb{R}^{d}\right)\right)},
$$

where $C_{1}=\tilde{K}\|\| c_{0}\left\|_{\infty}\left(1+T\|v\|_{\infty}\right) \sup _{t \leq T}\right\| \nabla u\left(t, X_{n, h}(t)\right)\left\|_{L^{2}(\Theta)}\right\|_{L^{2}(\Omega)}$, which is finite thanks to Lemma 3.1. To bound $\mathbb{E}_{\omega}\left[J_{2}\right]$, let $\chi(s, x):=\left(v_{N}\left(X_{n, h}\left(t_{l}\right)\right)-v_{N}(x)\right) \nabla u(s, x)$. Since $v_{N} \in \mathcal{C}_{b}^{2}\left(\mathbb{R}^{d}\right)$ and $u \in \mathcal{C}_{\text {pol }}^{1,4}\left(\left[0, T\left[\times \mathbb{R}^{d}\right)\right.\right.$, 
$\chi \in \mathcal{C}_{\text {pol }}^{1,2}\left(\left[0, T\left[\times \mathbb{R}^{d}\right)\right.\right.$. By Itô's formula, the derivative of $\varphi(s):=\mathbb{E}_{\theta}\left[\chi\left(s, X_{n, h}(s)\right)\right]$ in $\left(t_{l} ; t_{l+1}[\right.$ is given by:

$$
\frac{d \varphi}{d s}(s)=\mathbb{E}_{\theta}\left[\partial_{s} \chi\left(s, X_{n, h}(s)\right)+v_{N}\left(X_{n, h}\left(t_{l}\right)\right) \nabla \chi\left(s, X_{n, h}(s)\right)+D_{m} \triangle \chi\left(s, X_{n, h}(s)\right)\right] .
$$

Thanks to Lemma 3.1 the term $\mathbb{E}_{\omega}\left[\frac{d \varphi}{d s}\right]$ is bounded in $] t_{l} ; t_{l+1}\left[\right.$. Moreover, $\varphi\left(t_{l}\right)=0$, then, there exists $C_{2}>0$ such that $\mathbb{E}_{\omega}[\varphi(s)] \leq C_{2}\left(s-t_{l}\right)$, for $t_{l}<s<t_{l+1}$. Then, since $\mathbb{E}_{\omega}\left[J_{2}\right]=\int_{t_{l}}^{t_{l+1}} \mathbb{E}_{\omega}[\varphi(s)] d s$, we get:

$$
\mathbb{E}_{\omega}\left[J_{2}\right] \leq \frac{C_{2}}{2} d t^{2} .
$$

We obtain a bound of $\mathbb{E}_{\omega}\left[\mathbb{E}_{\theta}\left[e_{l}\right]\right]$ by combining (33) with (34), then the total error by taking the sum over $l$.

\subsection{Global error on the mean spread and the mean dispersion}

Now, we prove the final result.

Theorem 3.1. Suppose that the velocity $v_{N, h}$ has the same regularity as $v_{N}$ in $\Gamma$. Then, there exists a constant $C$, independent of $M, h$ and the time step $d t$ such that the following estimation holds:

$$
|\mathcal{E} r(T)| \leq C(g, \phi)\left(\sqrt{\sum_{m=N+1}^{\infty} \lambda_{m}}+d t+h+\frac{1}{\sqrt{M}}+\eta^{-\tau}\right) .
$$

Proof: We split this error into five terms $|\mathcal{E} r(T)| \leq\left|\mathcal{E} r_{1}\right|+\left|\mathcal{E} r_{2}\right|+\left|\mathcal{E} r_{3}\right|+\left|\mathcal{E} r_{4}\right|+\left|\mathcal{E} r_{5}\right|$, where we define: $\mathcal{E} r_{1}=\mathbb{E}_{\omega}\left[\phi\left(\mathbb{E}_{\theta}\left[g\left(Y_{T}\right)\right]\right)-\phi\left(\mathbb{E}_{\theta}\left[g_{N}\left(Y_{T}\right)\right]\right)\right], \mathcal{E} r_{2}=\mathbb{E}_{\omega}\left[\phi\left(\mathbb{E}_{\theta}\left[g_{N}\left(Y_{T}\right)\right]\right)-\phi\left(\mathbb{E}_{\theta}\left[g_{N}\left(X_{T}\right)\right]\right)\right]$, $\mathcal{E} r_{3}=\mathbb{E}_{\omega}\left[\phi\left(\mathbb{E}_{\theta}\left[g_{N}\left(X_{T}\right)\right]\right)-\phi\left(\mathbb{E}_{\theta}\left[g_{N}\left(X_{n, h}(T)\right)\right]\right)\right], \mathcal{E} r_{4}=\mathbb{E}_{\omega}\left[\phi\left(\mathbb{E}_{\theta}\left[g_{N}\left(X_{n, h}(T)\right]\right)-\phi\left(\frac{1}{M} \sum_{k=1}^{M} g_{N}\left(X_{n, h}^{k}(T)\right)\right)\right]\right.$, $\mathcal{E} r_{5}=\mathbb{E}_{\omega}\left[\phi\left(\frac{1}{M} \sum_{k=1}^{M} g_{N}\left(X_{n, h}^{k}(T)\right)\right]-\mathcal{Q}_{\alpha}(w, N) \phi\left(\frac{1}{M} \sum_{k=1}^{M} g_{N}\left(X_{n, h}^{k}(T)\right)\right)\right.$. By Taylor expansion:

$\left|\mathcal{E} r_{1}\right| \leq\left\|\frac{d \phi}{d x}(\xi)\right\|_{L^{2}(\Omega)}\left\|\mathbb{E}_{\theta}\left[g\left(Y_{T}\right)-g_{N}\left(Y_{T}\right)\right]\right\|_{L^{2}(\Omega)}$, where $\xi=\mathbb{E}_{\theta}\left[s g\left(Y_{T}\right)+(1-s) g_{N}\left(Y_{T}\right)\right]$ and $0<s<1$. We have $\mathbb{E}_{\theta}\left[g\left(Y_{T}\right)-g_{N}\left(Y_{T}\right)\right]=\mathbb{E}_{\theta}\left[g_{1}\left(Y_{T}\right)\left(g_{2}\left(Y_{T}\right)-g_{2 N}\left(Y_{T}\right)\right)\right] \leq\left\|g_{1}\left(Y_{T}\right)\right\|_{L^{1}(\Theta)}\left\|g_{2}-g_{2 N}\right\|_{L^{\infty}\left(\mathbb{R}^{d}\right)}$. Then we conclude, $\left|\mathcal{E} r_{1}\right| \leq\left\|\frac{d \phi}{d x}(\xi)\right\|_{L^{2}(\Omega)}\left\|g_{1}\left(Y_{T}\right)\right\|_{L^{4}\left(\Omega ; L^{1}(\Theta)\right)}\left\|g_{2}-g_{2 N}\right\|_{L^{4}\left(\Omega ; L^{\infty}\left(\mathbb{R}^{d}\right)\right)}$. Thanks to Lemma 3.1, $\left\|\frac{d \phi}{d x}(\xi)\right\|_{L^{2}(\Omega)}$ and $\left\|g_{1}\left(Y_{T}\right)\right\|_{L^{4}\left(\Omega ; L^{1}(\Theta)\right)}$ are finite, so by $(24)$, there is $C_{1}$ which depends on $g$ and $T$ such that:

$$
\left|\mathcal{E} r_{1}\right| \leq C_{1}\left(\sum_{m>N} \lambda_{m}\right)^{\frac{1}{2}}
$$

We have $\left|\mathcal{E} r_{2}\right| \leq\left\|\frac{d \phi}{d x}(\tilde{\xi})\right\|_{L^{2}(\Omega)}\left\|\mathbb{E}_{\theta}\left[g_{N}\left(Y_{T}\right)-g_{N}\left(X_{T}\right)\right]\right\|_{L^{2}(\Omega)}$, where $\tilde{\xi}=\mathbb{E}_{\theta}\left[s g_{N}\left(Y_{T}\right)+(1-s) g_{N}\left(X_{T}\right)\right]$. Thanks to Lemma 3.1, $\left\|\frac{d \phi}{d x}(\tilde{\xi})\right\|_{L^{2}(\Omega)}$ is finite, then using Proposition 3.2, there is $C_{2}>0$, independent of $N$ such that:

$$
\left|\mathcal{E} r_{2}\right| \leq C_{2}\left(\sum_{m>N} \lambda_{m}\right)^{\frac{1}{2}}
$$

Similarly, $\left|\mathcal{E} r_{3}\right| \leq\left\|\frac{d \phi}{d x}(\hat{\xi})\right\|_{L^{2}(\Omega)}\left\|\mathbb{E}_{\theta}\left[g_{N}\left(X_{T}\right)-g_{N}\left(X_{n, h}(T)\right)\right]\right\|_{L^{2}(\Omega)}$, where $\hat{\xi}=\mathbb{E}_{\theta}\left[s g_{N}\left(X_{T}\right)+(1-s) g_{N}\left(X_{n, h}(T)\right)\right]$.

Thanks to Lemma 3.1, $\left\|\frac{d \phi}{d x}(\hat{\xi})\right\|_{L^{2}(\Omega)}$ is finite, then by Proposition 3.3 we get: $\left|\mathcal{E} r_{3}\right| \leq C_{3}(d t+h)$.

For each $y \in \Gamma$, the random variables $\left\{g_{N}\left(X_{n, h}^{k}(y, T)\right)\right\}_{k=1}^{M}$ are independent, identically distributed in $\Theta$.

Then, using the law of large numbers and Taylor's formula, the fourth term satisfies: $\left|\mathcal{E} r_{4}\right| \leq C_{4} \frac{1}{\sqrt{M}}$. 
Let $\psi$ defined by $\psi(y)=\phi\left(\frac{1}{M} \sum_{k=1}^{M} g_{N}\left(X_{n, h}^{k}(y, T)\right)\right)$. It is easy to check that $\psi$ is an analytic function on $\Gamma$ and has the same regularity as $v_{N, h}(y,$.$) on \Gamma$. Therefore, by the bound (20) we get:

$$
\left|\mathcal{E} r_{5}\right|=\left|\int_{\Gamma} \psi(y) \varrho(y) d y-\mathcal{Q}_{\alpha}(w, N) \psi\right| \leq C_{5}(N, \alpha) \eta^{-\tau} .
$$

\section{Conclusion}

This work proposed and analyzed an efficient approach to compute quantities of interest for solute transport in random porous media. Our approach combines a stochastic collocation and a probabilistic Lagrangian method. The random data is approximated by a truncated K-L expansion, so that the mean quantities of interest are approximated by a quadrature rule based on an anisotropic sparse grid. For each point of this grid, the flow model is solved using a mixed finite element method in the physical space and the advection-diffusion equation is solved by a probabilistic particle method. The error estimates derived in this work predict the convergence rate. In particular, if $\tau>1 / 2$ and $\eta$ is not too large, then the method is more efficient than a classical Monte Carlo sampling.

\section{REFERENCES}

[1] I.M. BABUŜKa, F. Nobile, And R. Tempone: "A stochastic collocation method for elliptic partial differential equations with random input data", SIAM J. Numer. Anal, 43(3):1005-1034, 2007

[2] A. Beaudoin, J.-R. de Dreuzy, J. ERhel And G. Pichot "Convergence analysis of macro spreading in $3 D$ heterogeneous porous media", ESAIM Proceedings. December 2013, Vol. 41, p. 59-76.

[3] Brezzi, F. Fortin, M., " Mixed and Hybrid Finite Element Methods". Springer-Verlag, New York, NY, 1991

[4] J. CHARRIER. "Numerical analysis of the advection-diffusion of a solute in random media". INRIA-Report, (2011).

[5] J. Charrier and A. Debussche. "Weak truncation error estimates for elliptic pdes with lognormal coefficients. Stochastic Partial Differential Equations : Analysis and Computations", 1:63-93, 2013.

[6] J. ChARRIER. "Numerical analysis of the advection-diffusion of a solute in random media", preprint 2013. Marseille University.

[7] J. Erhel, Z. Mghazli, M. Oumouni: "Numerical analysis of stochastic advection-diffusion equation via Karhunen-Loève expansion". In preparation.

[8] J. Erhel, Z. Mghazli, M. Oumouni: "Calcul de l'espérance de la solution d'une EDP stochastique unidimensionnelle à l'aide d'une base réduite", C.R.Acad.Sci.Paris, Ser. I, 349 (2011), 861-865.

[9] Philipp Frauenfelder, Christoph Schwab, and Radu Alexandru Todor. "Finite elements for elliptic problems with stochastic coefficients". Comput. Methods Appl. Mech. Engrg., 194(2-5):205-228, 2005.

[10] Ioannis Karatzas and Steven E. Shreve. "Brownian motion and stochastic calculus", volume 113 of Graduate Texts in Mathematics. Springer-Verlag, New York, second edition, 1991.

[11] DentZ, M. Kinzelbach, H. Attinger, S. and W. Kinzelbach. "Temporal behavior of a solute cloud in a heterogeneous porous medium": 1. Point-like injection, Water Resources Research, 36 (12), pp. 3591-3604

[12] J.-R. de Dreuzy, A. Beaudoin, and J. Erhel. " Asymptotic dispersion in 2D heterogeneous porous media determined by parallel numerical simulations. Water Resource Research, 43 ( W10439, doi:10.1029/2006WR005394), 2007.

[13] Gelhar, L. "Stochastic Subsurface Hydrology", Engelwood Cliffs, New Jersey (1993).

[14] Ioannis Karatzas and Steven E. Shreve. "Brownian motion and stochastic calculus", volume 113 of Graduate Texts in Mathematics. Springer-Verlag, New York, second edition, 1991.

[15] A. Lunardi. " Analytic semigroups and optimal regularity in parabolic problems. Progress in Nonlinear Differential Equations and their Applications," 16. Birkhäuser Verlag, Basel, 1995.

[16] H. Schwarze, U. Jaekel And H. Vereecken. "Estimation of Macrodispersion by Different Approximation Methods for Flow and Transport in Randomly Heterogeneous Media". Transport in Porous Media 43: 265-287, 2001.

[17] R. Mikulevicius and E. Platen. " Rate of convergence of the Euler approximation for diffusion processes". Math. Nachr., 151:233-239, 1991.

[18] F. Nobile, R. Tempone, and C.Webster: "An anisotropic sparse grid stochastic collocation method for elliptic partial differential equations with random input data", SIAM J. Numer. Anal., 2008, vol. 46/5, pp. 2411-2442.

[19] M. Oumouni, "Analyse numérique de méthodes performantes pour les EDP stochastiques modélisant l'écoulement et le transport en milieux poreux". PhD thesis, Rennes1 University \& Ibn Tofaïl University, 2013.

[20] S. Smolyak: "Quadrature and Interpolation Formulas for Tensor Products of Certain Classes of Functions, Doklady Akademii Nauk SSSR, Volume 4, 1963, pages 240-243. 